\title{
Pacific
}

Journal of

Mathematics

\section{CURVATURES OF SPHERES IN HILBERT GEOMETRY}

AleXANDER BorisenKo AND EUGENE OLIN 


\title{
CURVATURES OF SPHERES IN HILBERT GEOMETRY
}

\author{
Alexander Borisenko And Eugene Olin
}

\begin{abstract}
We prove that the normal curvatures of hyperspheres, the Rund curvature, and the Finsler curvature of circles in Hilbert geometry tend to 1 as the radii tend to infinity.
\end{abstract}

\section{Introduction}

A smooth connected manifold $M^{n}$ is called a Finsler manifold [Bao et al. 2000] if there is a smooth positively homogeneous function $F: T M^{n} \rightarrow[0, \infty)$ on the coordinates in tangent spaces such that the symmetric bilinear form

$$
\boldsymbol{g}_{y}(u, v)=g_{i j}(x, y) u^{i} v^{j}: T_{x} M^{n} \times T_{x} M^{n} \rightarrow \mathbb{R}
$$

is positively definite for each pair $(x, y) \in T M^{n}$, where $g_{i j}(x, y)=\frac{1}{2}\left[F^{2}(x, y)\right]_{y^{i} y^{j}}$.

Consider a bounded open convex domain $U$ in $\mathbb{R}^{n}$ with the Euclidean norm $\|\cdot\|$, and let $\partial U$ be a $C^{3}$ hypersurface with positive normal curvatures. For a point $x \in U$ and a tangent vector $y \in T_{x} U=\mathbb{R}^{n}$, let $x_{-}$and $x_{+}$be the intersection points of the rays $x+\mathbb{R}_{-} y$ and $x+\mathbb{R}_{+} y$ with absolute $\partial U$. Then the Hilbert metric is defined as follows:

$$
F(x, y)=\frac{1}{2}(\Theta(x, y)+\Theta(x,-y)),
$$

where

$$
\Theta(x, y)=\|y\| \frac{1}{\left\|x-x_{+}\right\|}, \quad \Theta(x,-y)=\|y\| \frac{1}{\left\|x-x_{-}\right\|}
$$

are called the Funk metrics on $U$.

Hilbert geometries are the generalizations of Klein's model of the hyperbolic geometry. Hilbert geometries are also Finsler spaces of constant negative flag curvature -1 [Bao et al. 2000]. The Hilbert metric is invariant under projective transformations of $\mathbb{R}^{n}$ leaving $U$ bounded.

B. Colbois and P. Verovic [2002] proved that the Hilbert metric is asymptotically Riemannian at infinity. That means that in a given Hilbert geometry the unit sphere

MSC2010: 53B40, 53C60.

Keywords: Hilbert geometry, Finsler geometry, spheres, norman curvature, Rund curvature, Finsler curvature. 
of the norm $F(x, \cdot)$ approaches the ellipsoid in $C^{0}$ topology as the point $x$ tends to $\partial U$.

Unlike the Riemannian geometry, in the Finsler geometry there are several definitions of the curvature of a curve.

The normal curvature of a hypersurface in a Finsler space is defined as follows [Shen 2001]. Let $\varphi: N \rightarrow M^{n}$ be a hypersurface in a Finsler manifold $M^{n}$. A vector $\boldsymbol{n} \in T_{\varphi(x)} M^{n}$ is called a normal vector to $N$ at the point $x \in N$ if $\boldsymbol{g}_{\boldsymbol{n}}(y, \boldsymbol{n})=0$ for all $y \in T_{x} N$. The normal curvature $\boldsymbol{k}_{\boldsymbol{n}}$ at the point $x \in N$ in a direction $y \in T_{x} N$ is defined as

$$
\boldsymbol{k}_{\boldsymbol{n}}=\boldsymbol{g}_{\boldsymbol{n}}\left(\left.\nabla_{\dot{c}(s)} \dot{c}(s)\right|_{s=0}, \boldsymbol{n}\right),
$$

where $\dot{c}(0)=y, c(s)$ is a geodesic in the induced connection on $N$, and $\boldsymbol{n}$ is the chosen unit normal vector.

For a curve $c(s)$ parametrized by its arc length in $M^{n}$, it is possible to define two more curvatures.

The Finsler curvature of $c(s)$ [Finsler 1951; Rund 1959] is defined as

$$
\boldsymbol{k}_{F}(c(s))=\sqrt{\boldsymbol{g}_{\dot{c}(s)}\left(\nabla_{\dot{c}(s)} \dot{c}(s), \nabla_{\dot{c}(s)} \dot{c}(s)\right)} .
$$

The Rund curvature of $c(s)$ [Rund 1959] is defined as

$$
\boldsymbol{k}_{R}(c(s))=\sqrt{\boldsymbol{g}_{\nabla_{\dot{c}(s)} \dot{c}(s)}\left(\nabla_{\dot{c}(s)} \dot{c}(s), \nabla_{\dot{c}(s)} \dot{c}(s)\right)} .
$$

It is well-known that the normal curvatures of hyperspheres in the hyperbolic space $\mathbb{U}^{n}$ are equal to $\operatorname{coth}(r)$ and tend to 1 as the radius $r$ tends to infinity. We prove the same property for the Hilbert geometry.

Theorem 1.1. The normal curvature, the Rund curvature, and the Finsler curvature of the circles centered at the same point in the 2-dimensional Hilbert geometry tend to 1 as their radii tend to infinity, uniformly at the point of the circle.

Theorem 1.2. The normal curvatures of the hyperspheres centered at the same point tend to 1 as their radii tend to infinity, uniformly at the point of the hypersphere and in the tangent vector at this point of the hypersphere.

This can be interpreted as meaning that the Hilbert metric tends to the Riemannian metric of the hyperbolic space in $C^{2}$-topology.

\section{The choice of the coordinate system}

Consider the Hilbert geometry based on a two-dimensional domain $U$ in the Euclidean plane. Fix a point $o$ in the domain $U$ and a point $p \in \partial U$. Since $\partial U$ is a convex curve, it admits the polar representation $\omega(\varphi)$ from the point $o$ such that the point $p$ corresponds to $\varphi=0$. 
Choose the coordinate system on the plane with the origin $O$ at the point $p$; let the axis $x_{2}$ be orthogonal to $\partial U$ at $p, x_{1}$ be tangent to $\partial U$ at $p$, and $U-\{p\}$ lie in the half-plane $x_{2}>0$.

In this section we will construct a projective transformation $P$ of the plane that sends $U$ to $\hat{U}$ and has the following properties:

(1) $P(p)=p$.

(2) The vector $u=(0,1)$ is orthogonal to $\partial \hat{U}$ at the point $p$.

(3) The tangent line to $\partial \hat{U}$ at the point $p$ is parallel to the tangent line to $\partial \hat{U}$ at the point corresponding to $\varphi=\pi$.

(4) $\partial \hat{U}$ is the graph of the function $x_{2}=\hat{f}\left(x_{1}\right)$ such that $\hat{f}(0)=0, \hat{f}^{\prime}(0)=0$, and $\hat{f}^{\prime \prime}(0)=\frac{1}{2}$ in the neighborhood of $p$.

We are going to give the explicit expression for this transformation and show that after this transformation the curvature of $\partial \hat{U}$ and the derivatives of $f$ remain uniformly bounded.

The next lemma gives the upper bound on the angle between the radial and normal direction to the convex curve.

Lemma 2.1 [Borisenko 2002]. Let $\gamma$ be a closed embedded curve in the Euclidean plane whose curvature is greater than or equal to $k$. Let o be a point in the interior of the set bounded by $\gamma, \omega_{0}$ the distance from o to $\gamma$, and $\varphi$ the angle between the outer normal vector at the point $p \in \gamma$ and the vector op. Then

$$
\cos \angle\left(u_{m}, N(m)\right) \geqslant \omega_{0} k .
$$

Denote by $k$ and $K$ the minimum and maximum of the curvatures of $\partial U$. Also, $\omega_{0}=\min _{\varphi} \omega(\varphi), \omega_{1}=\max _{\varphi} \omega(\varphi)$.

Let the length of the chord of $U$ in the direction $u$ equal $H$, the distance from $o$ to the origin equal $\omega_{u}, \omega_{0} \leqslant \omega_{u} \leqslant \omega_{1}$, and the angle between $u$ and $x_{2}$ equal $\alpha$.

Step 1. Construct an affine transformation that makes the vector $\overrightarrow{o O}$ parallel to $x_{2}$. This transformation sends the points $(0,0)$ and $(1,0)$ to themselves, the point ( $H \sin \alpha, H \cos \alpha) \in \partial U$ to the point $(0, H)$, and has the expression:

$$
\tilde{x}_{1}=x_{1}-\tan \alpha x_{2}, \quad \tilde{x}_{2}=\frac{x_{2}}{\cos \alpha} .
$$

Denote the image of $U$ as $\tilde{U}$. The point $o$ now has the coordinates $\left(0, \omega_{u}\right)$. Denote by $\tilde{k}$ the minimum of the curvature of $\partial \tilde{U}$ in the $\left(\tilde{x}_{1}, \tilde{x}_{2}\right)$ coordinate system, and by $\tilde{\omega}_{0}$ denote the distance from the point $\left(0, \omega_{u}\right)$ to $\partial \tilde{U}$. Note that the eigenvalues of the transformation (6) are equal to 1 and $1 / \cos \alpha$. Hence

$$
\omega_{0} \leqslant \tilde{\omega}_{0} \leqslant \frac{1}{\cos \alpha} \omega_{0} .
$$


Lemma 2.1 then implies that the curvature of $\partial \tilde{U}$ remains bounded and separated from zero.

Step 2. Construct the transformation such that the tangent line

$$
\tilde{x}_{2}=-\tan \beta \tilde{x}_{1}+H
$$

to $\partial \tilde{U}$ at the point $(0, H)$ will be parallel to the axis $\tilde{x}_{1}$, where $\beta$ is the angle between $\tilde{x}_{2}$ and the normal vector to $\partial \tilde{U}$ at $(0, H)$. This transformation has the expression

$$
\bar{x}_{1}=\frac{H \tilde{x}_{1}}{H-\tan \beta \tilde{x}_{1}}, \quad \bar{x}_{2}=\frac{H \tilde{x}_{2}}{H-\tan \beta \tilde{x}_{1}} .
$$

Denote the image of $\tilde{U}$ as $\bar{U}$.

We can estimate the angle $|\tan \beta|$. Using Lemma 2.1, we have

$$
0 \leqslant|\tan \beta| \leqslant \sqrt{\frac{1}{\left(\tilde{k}^{2} \tilde{\omega}_{0}^{2}\right)}-1 .}
$$

Estimate the curvature $\partial \bar{U}$. Let the curve $\partial \tilde{U}$ be given in the parametric form $r(t)=\left(\tilde{x}_{1}(t), \tilde{x}_{2}(t)\right)$. Then $\partial \bar{U}$ has the parametrization

$$
\bar{r}(t)=\frac{H r(t)}{H-\tan \beta \tilde{x}_{1}(t)} .
$$

Differentiating leads to

$$
\begin{aligned}
\bar{r}^{\prime}(t)= & \frac{H r^{\prime}(t)}{H-\tan \beta \tilde{x}_{1}(t)}+\frac{H r(t) \tan \beta \tilde{x}_{1}^{\prime}(t)}{\left(H-\tan \beta \tilde{x}_{1}(t)\right)^{2}}, \\
\bar{r}^{\prime \prime}(t)= & \frac{2 H \tan \beta r^{\prime}(t) \tilde{x}_{1}^{\prime}(t)}{\left(H-\tan \beta \tilde{x}_{1}(t)\right)^{2}}+\frac{2 H r(t) \tan ^{2} \beta \tilde{x}_{1}^{\prime}(t)^{2}}{\left(H-\tan \beta \tilde{x}_{1}(t)\right)^{3}} \\
& +\frac{H r^{\prime \prime}(t)}{H-\tan \beta \tilde{x}_{1}(t)}+\frac{H r(t) \tan \beta \tilde{x}_{1}^{\prime \prime}(t)}{\left(H-\tan \beta \tilde{x}_{1}(t)\right)^{2}} .
\end{aligned}
$$

The strict convexity of $\partial \tilde{U}$ implies that $H-\tan \beta \tilde{x}_{1}(t) \geqslant$ const $>0$ for each $t$. This and the compactness argument leads to the maximum of the curvature of $\partial \bar{U}$ being bounded from above for some constant.

If the curve $\partial \tilde{U}$ is the graph $\tilde{x}_{2}=f\left(\tilde{x}_{1}\right)$ and $f(0)=f^{\prime}(0)=0$, then its curvature at the point $(0,0)$ after the transformation (8) will not change. Indeed,

$$
\begin{aligned}
\tilde{x}_{1}^{\prime}(t)^{2}+ & \tilde{x}_{2}^{\prime}(t)^{2} \\
& =\left(\frac{H t \tan \beta}{(H-t \tan \beta)^{2}}+\frac{H}{H-t \tan \beta}\right)^{2}+\left(\frac{H \tan \beta f(t)}{(H-t \tan \beta)^{2}}+\frac{H f^{\prime}(t)}{H-t \tan \beta}\right)^{2},
\end{aligned}
$$




$$
\begin{aligned}
& \tilde{x}_{1}^{\prime}(t) \tilde{x}_{2}^{\prime \prime}(t)-\tilde{x}_{1}^{\prime \prime}(t) \tilde{x}_{2}^{\prime}(t) \\
& =-\left(\frac{2 H t \tan ^{2} \beta}{(H-t \tan \beta)^{3}}+\frac{2 H \tan \beta}{(H-t \tan \beta)^{2}}\right)\left(\frac{H \tan \beta f(t)}{(H-t \tan \beta)^{2}}+\frac{H f^{\prime}(t)}{H-t \tan \beta}\right) \\
& +\left(\frac{H t \tan \beta}{(H-t \tan \beta)^{2}}+\frac{H}{H-t \tan \beta}\right)\left(\frac{2 H \tan ^{2} \beta f(t)}{(H-t \tan \beta)^{3}}+\frac{2 H \tan \beta f^{\prime}(t)}{(H-t \tan \beta)^{2}}+\frac{H f^{\prime \prime}(t)}{H-t \tan \beta}\right) .
\end{aligned}
$$

We obtain the claim after substituting the equalities $f(0)=f^{\prime}(0)=0$. So the curvature of $\partial \bar{U}$ at the origin is still separated from zero.

Step 3. Construct a transformation such that the distance from $\left(0, \omega_{u}\right)$ to the origin is equal to 1 and the curvature of $\partial \bar{U}$ at the origin is equal to $\frac{1}{2}$. This transformation has the expression:

$$
\hat{x}_{1}=\frac{\bar{x}_{1}}{\omega_{u}}, \quad \hat{x}_{2}=\frac{\bar{x}_{2}}{2 \omega_{u}^{2} \bar{k}(0)} .
$$

Denote the image of $\bar{U}$ as $\hat{U}$. It is obvious that the curvature of $\partial \hat{U}$ remains bounded.

The announced transformation $P$ is the composition of the transformations (6), (8), and (10), and the following proposition holds:

Proposition 2.2. There exists a constant $C_{0}$ depending on $U$ such that the curvature of $P(\partial U)$ is bounded from above by $C_{0}$.

Let $\partial U$ be the graph of the function $x_{2}=f\left(x_{1}\right)$ in the initial coordinate system. After the transformation $\mathrm{P}, P(\partial U)$ can be considered the graph of the function $x_{2}=\hat{f}\left(x_{1}\right)$ such that $\hat{f}(0)=0, \hat{f}^{\prime}(0)=0$, and $\hat{f}^{\prime \prime}(0)=\frac{1}{2}$ in the neighborhood of $p$.

Finally, estimate the third derivative $\hat{f}^{\prime \prime \prime}(0)$. Evidently, under the affine transformations (6) and (10) the third derivative remains bounded. We only need to control $f^{\prime \prime \prime}(0)$ at Step 2.

So let the curve $\partial \tilde{U}$ be the graph $\tilde{x}_{2}=\tilde{f}\left(\tilde{x}_{1}\right)$ and after the transformation (8) we obtain the graph $\bar{f}$. The rules for differentiation lead to

$$
\bar{f}^{\prime \prime \prime}(0)=\tilde{f}^{\prime \prime \prime}(0)-\frac{\tan \beta \tilde{k}(0)}{H} .
$$

As $\partial U$ is the compact curve, we obtain:

Proposition 2.3. There exist constants $C_{1}, C_{2}$ depending on $U$, such that

$$
C_{1} \leqslant \hat{f}^{\prime \prime \prime}(0) \leqslant C_{2} \text {. }
$$

Analogously we can estimate all higher derivatives. 
The Hilbert metrics for the domains $U$ and $\hat{U}$ are isometric. Therefore, without loss of generality, we will consider the Hilbert metric for the domain $\hat{U}$ and will denote $\hat{U}$ by $U$.

\section{Series expansions for the metric tensor of the Hilbert metric}

From the decomposition of the Hilbert metric through the Funk metrics (1), we conclude

$$
\begin{aligned}
g_{i j}(x, y)= & \mathrm{F}(x, y) \mathrm{F}_{y^{i} y^{j}}(x, y)+\mathrm{F}_{y^{i}}(x, y) \mathrm{F}_{y^{j}}(x, y) \\
= & \frac{1}{2} \mathrm{~F}(x, y)\left(\Theta_{y^{i} y^{j}}(x, y)+\Theta_{y^{i} y^{j}}(x,-y)\right) \\
& \quad+\frac{1}{4}\left(\Theta_{y^{i}}(x, y)-\Theta_{y^{i}}(x,-y)\right)\left(\Theta_{y^{j}}(x, y)-\Theta_{y^{j}}(x,-y)\right) .
\end{aligned}
$$

Okada's lemma [Shen 2001] for Funk metrics gives the expression of the derivatives of $\Theta(x, y)$ with respect to the coordinates on tangent spaces through the derivatives with respect to the coordinates on $U$ :

$$
\Theta(x, y)_{x^{k}}=\Theta(x, y) \Theta(x, y)_{y^{k}} .
$$

Using this lemma, we can write:

$$
\begin{aligned}
g_{i j}(x, y)= & \frac{1}{2} \mathrm{~F}(x, y) \frac{\Theta_{x^{i} x^{j}}(x, y) \Theta(x, y)-2 \Theta_{x_{i}}(x, y) \Theta_{x_{j}}(x, y)}{\Theta(x, y)^{3}} \\
& +\frac{1}{2} \mathrm{~F}(x, y) \frac{\Theta_{x^{i} x^{j}}(x,-y) \Theta(x,-y)-2 \Theta_{x_{i}}(x,-y) \Theta_{x_{j}}(x,-y)}{\Theta(x,-y)^{3}} \\
& +\frac{1}{4}\left(\frac{\Theta_{x^{i}}(x, y)}{\Theta(x, y)}-\frac{\Theta_{x^{i}}(x,-y)}{\Theta(x,-y)}\right)\left(\frac{\Theta_{x^{j}}(x, y)}{\Theta(x, y)}-\frac{\Theta_{x^{j}}(x,-y)}{\Theta(x,-y)}\right) .
\end{aligned}
$$

For convenience we will use lower indices $x_{i}$ for coordinates. Let $\mathrm{F}\left(x_{1}, x_{2}, y_{1}, y_{2}\right)$ be a two-dimensional Hilbert metric and $\Theta\left(x_{1}, x_{2}, y_{1}, y_{2}\right)$ the corresponding Funk metric. Assume that the point $\left(x_{1}, x_{2}\right)$ is sufficiently close to $\partial U$. Then we can express $\partial U$ as the graph $x_{2}=f\left(x_{1}\right)$ such that $f(0)=0, f^{\prime}(0)=0$, and $f^{\prime \prime}(0)=\frac{1}{2}$. Consider a point $\left(x_{1}, x_{2}\right)$ above the graph $x_{2}=f\left(x_{1}\right)$. Denote by $r\left(x_{1}, x_{2}, y_{1}, y_{2}\right)$ the distance between the point $\left(x_{1}, x_{2}\right)$ and the intersection point of the line passing trough $\left(x_{1}, x_{2}\right)$ in the direction $\left(y_{1}, y_{2}\right)$ with the curve $x_{2}=f\left(x_{1}\right)$. Then

$$
\Theta\left(x_{1}, x_{2}, y_{1}, y_{2}\right)=\sqrt{y_{1}^{2}+y_{2}^{2}} r\left(x_{1}, x_{2}, y_{1}, y_{2}\right)^{-1} .
$$

Now we obtain the derivatives of $r\left(x_{1}, x_{2}, y_{1}, y_{2}\right)$ on $x_{1}, x_{2}$. The parameter $t\left(x_{1}, x_{2}, y_{1}, y_{2}\right)$ corresponding to the intersection points of the curve $x_{2}=f\left(x_{1}\right)$ with the line

$$
x_{1}(t)=x_{1}+t y_{1}, \quad x_{2}(t)=x_{2}+t y_{2}
$$


satisfies the functional equation

$$
x_{2}+t y_{2}=f\left(x_{1}+t\left(x_{1}, x_{2}, y_{1}, y_{2}\right) y_{1}\right) \text {. }
$$

Differentiate (14) on $x_{1}, x_{2}$ :

$$
t_{x_{1}} y_{2}=f^{\prime}\left(x_{1}+t y_{1}\right)\left(1+t_{x_{1}} y_{1}\right), \quad 1+t_{x_{2}} y_{2}=f^{\prime}\left(x_{1}+t y_{1}\right) t_{x_{2}} y_{1} .
$$

We obtain the explicit expressions for $t_{x_{1}}, t_{x_{2}}$ :

$$
t_{x_{1}}=\frac{f^{\prime}\left(x_{1}+t y_{1}\right)}{y_{2}-y_{1} f^{\prime}\left(x_{1}+t y_{1}\right)}, \quad t_{x_{2}}=\frac{1}{y_{1} f^{\prime}\left(x_{1}+t y_{1}\right)-y_{2}} .
$$

Differentiating (15) leads to

$$
\begin{aligned}
& y_{2} t_{x_{1} x_{1}}=f^{\prime \prime}\left(x_{1}+t y_{1}\right)\left(1+y_{1} t_{x_{1}}\right)^{2}+f^{\prime}\left(x_{1}+t y_{1}\right) y_{1} t_{x_{1} x_{1}}, \\
& y_{2} t_{x_{1} x_{2}}=f^{\prime \prime}\left(x_{1}+t y_{1}\right)\left(1+y_{1} t_{x_{1}}\right) y_{2} t_{x_{2}}+f^{\prime}\left(x_{1}+t y_{1}\right) y_{1} t_{x_{1} x_{2}}, \\
& y_{2} t_{x_{2} x_{2}}=f^{\prime \prime}\left(x_{1}+t y_{1}\right)\left(y_{1} t_{x_{2}}\right)^{2}+f^{\prime}\left(x_{1}+t y_{1}\right) y_{1} t_{x_{2} x_{2}} .
\end{aligned}
$$

We obtain the expressions for the second derivatives of $t$ :

$$
\begin{aligned}
t_{x_{1} x_{1}} & =\frac{f^{\prime \prime}\left(x_{1}+t y_{1}\right)\left(1+y_{1} t_{x_{1}}\right)^{2}}{y_{2}-y_{1} f^{\prime}\left(x_{1}+t y_{1}\right)}, \\
t_{x_{1} x_{2}} & =\frac{f^{\prime \prime}\left(x_{1}+t y_{1}\right)\left(1+y_{1} t_{x_{1}}\right) y_{1} t_{x_{2}}}{y_{2}-y_{1} f^{\prime}\left(x_{1}+t y_{1}\right)}, \\
t_{x_{2} x_{2}} & =\frac{f^{\prime \prime}\left(x_{1}+t y_{1}\right)\left(y_{1} t_{x_{2}}\right)^{2}}{y_{2}-y_{1} f^{\prime}\left(x_{1}+t y_{1}\right)} .
\end{aligned}
$$

We need the derivatives of $r\left(x_{1}, x_{2}, y_{1}, y_{2}\right)$. By definition,

$$
r\left(x_{1}, x_{2}, y_{1}, y_{2}\right)=\sqrt{\left(y_{1} t\right)^{2}+\left(y_{2} t\right)^{2}}=\sqrt{y_{1}^{2}+y_{2}^{2}} t\left(x_{1}, x_{2}, y_{1}, y_{2}\right) .
$$

Hence $r_{x_{k}}=t_{x_{k}}$ and $r_{x_{k} x_{l}}=t_{x_{k} x_{l}}$.

Now it is possible to calculate the derivatives of the Funk metric. Formula (13) implies

$$
\Theta_{x_{k}}=-\sqrt{y_{1}^{2}+y_{2}^{2}} \frac{r_{x_{k}}}{r^{2}} .
$$

After differentiating (19), we obtain

$$
\Theta_{x_{k} x_{l}}=-\sqrt{y_{1}^{2}+y_{2}^{2}} \frac{r_{x_{k} x_{l}} r^{2}-2 r r_{x_{l}} r_{x_{k}}}{r^{4}}=\sqrt{y_{1}^{2}+y_{2}^{2}}\left(2 \Theta^{3} r_{x_{k}} r_{x_{l}}-\Theta^{2} r_{x_{k} x_{l}}\right) .
$$

Finally, from (12) it is possible to obtain the coefficients of the metric tensor. We will need the values of $g_{i j}\left(x_{1}, x_{2}, y_{1}, y_{2}\right)$ at the points $\left(x_{1}, x_{2}\right)=\left(0, x_{2}\right)$. 
3.1. Expansions for $\boldsymbol{g}_{\boldsymbol{i j}}\left(\mathbf{0}, \boldsymbol{x}_{\mathbf{2}}, \mathbf{1}, \mathbf{0}\right)$. Note that the strict convexity of $\partial U$ implies that $f^{\prime}\left(t\left(x_{1}, x_{2}\right)\right) \neq 0$ for $t\left(x_{1}, x_{2}\right) \neq 0$. Then from (16) we deduce

$$
\begin{aligned}
& t_{x_{1}}\left(0, x_{2}, 1,0\right)=-1, \\
& t_{x_{2}}\left(0, x_{2}, 1,0\right)=\frac{1}{f^{\prime}\left(t\left(0, x_{2}, 1,0\right)\right)},
\end{aligned}
$$

and from (18)

$$
\begin{aligned}
& t_{x_{1} x_{1}}\left(0, x_{2}, 1,0\right)=t_{x_{1} x_{2}}\left(0, x_{2}, 1,0\right)=0, \\
& t_{x_{2} x_{2}}\left(0, x_{2}, 1,0\right)=-\frac{f^{\prime \prime}\left(t\left(0, x_{2}, 1,0\right)\right)}{f^{\prime}\left(t\left(0, x_{2}, 1,0\right)\right)^{3}} .
\end{aligned}
$$

Expanding the functional equation (14) in a power series with respect to $t$ as $x_{2} \rightarrow 0$, we find the expansions of $t\left(0, x_{2}, 1,0\right)$.

$$
x_{2}=\frac{1}{4} t^{2}+\frac{1}{6} f^{\prime \prime \prime}(0) t^{3}+O\left(t^{4}\right) .
$$

We will find $t$ in expanded form

$$
t=A+B \sqrt{x_{2}}+C x_{2}+D x_{2}^{3 / 2}+O\left(x_{2}^{2}\right)
$$

After substituting (26) into (25) and transposing all members in the left side, we obtain the following system of equations:

$$
\begin{aligned}
& 3 A^{2}+2 A^{3} f^{\prime \prime \prime}(0)+\left(6 A B+6 A^{2} f^{\prime \prime \prime}(0) B\right) \sqrt{x_{2}} \\
& +\left(-12+3 B^{2}+6 A f^{\prime \prime \prime}(0) B^{2}+6 A C+6 A^{2} f^{\prime \prime \prime}(0) C\right) x_{2}+\left(2 f^{\prime \prime \prime}(0) B^{2}\right. \\
& \left.\quad+6 B C+12 A f^{\prime \prime \prime}(0) B C+6 A D+6 A^{2} f^{\prime \prime \prime}(0) D\right) x_{2}^{3 / 2}+O\left(x_{2}^{2}\right)=0 .
\end{aligned}
$$

Choose the coefficients $A, B, C$, and $D$ so that the left side of (27) is $O\left(x_{2}^{2}\right)$. Equating the coefficients under the powers of $x_{2}$ to zero we obtain two expansions for $t$ which correspond to the directions $(1,0)$ and $(-1,0)$.

$$
t\left(0, x_{2}, \pm 1,0\right)= \pm 2 \sqrt{x_{2}}-\frac{4}{3} f^{\prime \prime \prime}(0) x_{2}+O\left(x_{2}^{2}\right) .
$$

In our case $r=t$, so we get

$$
r\left(0, x_{2}, 1,0\right)=2 \sqrt{x_{2}}-\frac{4}{3} f^{\prime \prime \prime}(0) x_{2}+O\left(x_{2}^{2}\right) .
$$

Later on, all power series will be considered in the neighborhood of 0 . The series expansion for the metric $\mathrm{F}$ is 


$$
\begin{aligned}
\mathrm{F}\left(0, x_{2}, 1,0\right) & =\frac{1}{2}\left(\frac{1}{r\left(0, x_{2}, 1,0\right)}+\frac{1}{r\left(0, x_{2},-1,0\right)}\right) \\
& =\frac{1}{2}\left(\frac{1}{2 \sqrt{x_{2}}-\frac{4}{3} f^{\prime \prime \prime}(0) x_{2}+O\left(x_{2}^{2}\right)}+\frac{1}{2 \sqrt{x_{2}}+\frac{4}{3} f^{\prime \prime \prime}(0) x_{2}+O\left(x_{2}^{2}\right)}\right) \\
& =\frac{9}{\sqrt{x_{2}}\left(18-8 f^{\prime \prime \prime}(0)^{2} x_{2}\right)+O\left(x_{2}^{3 / 2}\right)} . \\
& \mathrm{F}\left(0, x_{2}, 1,0\right)=\frac{1}{2 \sqrt{x_{2}}}+\frac{2 f^{\prime \prime \prime}(0)^{2}}{9} \sqrt{x_{2}}+O\left(x_{2}^{3 / 2}\right) .
\end{aligned}
$$

We will also need the difference:

$$
\begin{aligned}
\Theta\left(0, x_{2}, 1,0\right)-\Theta\left(0, x_{2},-1,0\right) & =\frac{1}{r\left(0, x_{2}, 1,0\right)}-\frac{1}{r\left(0, x_{2},-1,0\right)} \\
& =\frac{-6 f^{\prime \prime \prime}(0)+O\left(x_{2}\right)}{4 f^{\prime \prime \prime}(0)^{2} x_{2}-9+O\left(x_{2}^{3 / 2}\right)} \\
& =\frac{2}{3} f^{\prime \prime \prime}(0)+O\left(x_{2}\right) .
\end{aligned}
$$

From (21), using $r_{x_{k}}=t_{x_{k}}$, we get

$$
r_{x_{1}}\left(0, x_{2}, 1,0\right)=-1 \text {. }
$$

Expand the denominator of (22) with respect to $t$ :

$$
\begin{aligned}
r_{x_{2}}\left(0, x_{2}, 1,0\right) & =\frac{1}{f^{\prime}\left(t\left(0, x_{2}, 1,0\right)\right.} \\
& =\frac{1}{f^{\prime \prime}(0) t\left(0, x_{2}, 1,0\right)+\frac{1}{2} f^{\prime \prime \prime}(0) t\left(0, x_{2}, 1,0\right)^{2}+O\left(t\left(0, x_{2}, 1,0\right)^{3}\right)} .
\end{aligned}
$$

Using the fact that $f^{\prime}(0)=0$ and $f^{\prime \prime}(0)=\frac{1}{2}$ and substituting the value of $t$ from (28), we obtain

$$
r_{x_{2}}\left(0, x_{2}, 1,0\right)=\frac{1}{\frac{1}{2}\left(2 \sqrt{x_{2}}-\frac{4}{3} f^{\prime \prime \prime}(0) x_{2}\right)+\frac{1}{2} f^{\prime \prime \prime}(0)\left(2 \sqrt{x_{2}}-\frac{4}{3} f^{\prime \prime \prime}(0) x_{2}\right)^{2}+O\left(x_{2}^{2}\right)} .
$$

$r_{x_{2}}\left(0, x_{2},-1,0\right)$ is analogous. Finally,

$$
r_{x_{2}}\left(0, x_{2}, 1,0\right)=\frac{1}{\sqrt{x_{2}}}-\frac{4 f^{\prime \prime \prime}(0)}{3}+\frac{40 f^{\prime \prime \prime}(0)^{2}}{9} \sqrt{x_{2}}+O\left(x_{2}\right) .
$$

The second derivative has the form $r_{x_{k} x_{l}}=t_{x_{k} x_{l}}$. From (23) we obtain

$$
r_{x_{1} x_{1}}\left(0, x_{2}, 1,0\right)=r_{x_{1} x_{2}}\left(0, x_{2}, 1,0\right)=0 .
$$

And (24) implies

$$
r_{x_{2} x_{2}}\left(0, x_{2}, 1,0\right)=-\frac{f^{\prime \prime}\left(t\left(0, x_{2}, 1,0\right)\right)}{f^{\prime}\left(t\left(0, x_{2}, 1,0\right)\right)^{3}} .
$$


We now expand the numerator and denominator in a series with respect to $t$ and use $f^{\prime}(0)=0, f^{\prime \prime}(0)=\frac{1}{2}$, and $(28)$ :

$$
\begin{aligned}
& r_{x_{2} x_{2}}\left(0, x_{2}, 1,0\right) \\
& =-\frac{\frac{1}{2}+f^{\prime \prime \prime}(0) t\left(0, x_{2}, 1,0\right)+\frac{1}{2} f^{(4)}(0) t\left(0, x_{2}, 1,0\right)^{2}+O\left(t^{3}\right)}{\left(f^{\prime \prime}(0) t\left(0, x_{2}, 1,0\right)+\frac{1}{2} f^{\prime \prime \prime}(0) t\left(0, x_{2}, 1,0\right)^{2}+O\left(t\left(0, x_{2}, 1,0\right)^{3}\right)\right)^{3}} \\
& =\frac{-\frac{1}{2}-2 f^{\prime \prime \prime}(0) \sqrt{x_{2}}+\left(\frac{4}{3} f^{\prime \prime \prime}(0)^{2}-4 f^{(4)}(0)\right) x_{2}+\frac{16}{3} f^{\prime \prime \prime}(0) f^{(4)}(0) x_{2}^{3 / 2}+O\left(x_{2}^{2}\right)}{x_{2}^{3 / 2}+4 f^{\prime \prime \prime}(0) x_{2}^{2}+O\left(x_{2}^{5 / 2}\right)}
\end{aligned}
$$

Thus

$$
r_{x_{2} x_{2}}\left(0, x_{2}, 1,0\right)=-\frac{1}{2 x_{2}^{3 / 2}}-\frac{2 f^{(4)}(0)}{\sqrt{x_{2}}}+O(1) .
$$

From (19), (29), (32) we find that

$$
\Theta_{x_{1}}\left(0, x_{2}, 1,0\right)=\frac{1}{\left(2 \sqrt{x_{2}}-\frac{4}{3} f^{\prime \prime \prime}(0) x_{2}+O\left(x_{2}^{2}\right)\right)^{2}} .
$$

Analogously, acting for the vector $(-1,0)$, we get

$$
\Theta_{x_{1}}\left(0, x_{2}, \pm 1,0\right)= \pm \frac{1}{4 x_{2}}+\frac{f^{\prime \prime \prime}(0)}{3 \sqrt{x_{2}}}+O(1) .
$$

From (29) and (33) we deduce

$$
\Theta_{x_{2}}\left(0, x_{2}, 1,0\right)=-\frac{1 / \sqrt{x_{2}}-4 f^{\prime \prime \prime}(0) / 3+\left(40 f^{\prime \prime \prime}(0) / 9\right) \sqrt{x_{2}}+O\left(x_{2}\right)}{\left(2 \sqrt{x_{2}}-\frac{4}{3} f^{\prime \prime \prime}(0) x_{2}+O\left(x_{2}^{2}\right)\right)^{2}},
$$

and finally,

$$
\Theta_{x_{2}}\left(0, x_{2}, \pm 1,0\right)=-\frac{1}{4 x_{2}^{3 / 2}}-\frac{f^{\prime \prime \prime}(0)^{2}}{\sqrt{x_{2}}}+O(1) .
$$

Using the formulae (20), (29), (33), and (35), we obtain the expression for the second derivatives of the Funk metric:

$$
\Theta_{x_{2} x_{2}}\left(0, x_{2}, \pm 1,0\right)=\frac{3}{8 x_{2}^{5 / 2}}+\frac{13 f^{\prime \prime \prime}(0)^{2}+3 f^{(4)}(0)}{6 x_{2}^{3 / 2}}+O\left(\frac{1}{x_{2}}\right) .
$$

Finally we can estimate the metric coefficients. From (13), (29), and (36) we get

$$
\frac{\Theta_{x_{1}}\left(0, x_{2}, 1,0\right)}{\Theta\left(0, x_{2}, 1,0\right)}-\frac{\Theta_{x_{1}}\left(0, x_{2},-1,0\right)}{\Theta\left(0, x_{2},-1,0\right)}=\frac{1}{\sqrt{x_{2}}}+\frac{4 f^{\prime \prime \prime}(0)^{2}}{9} \sqrt{x_{2}}+O\left(x_{2}^{3 / 2}\right) .
$$


It follows from (13), (29), and (37) that

$$
\frac{\Theta_{x_{2}}\left(0, x_{2}, 1,0\right)}{\Theta\left(0, x_{2}, 1,0\right)}-\frac{\Theta_{x_{2}}\left(0, x_{2},-1,0\right)}{\Theta\left(0, x_{2},-1,0\right)}=\frac{2 f^{\prime \prime \prime}(0)}{3 \sqrt{x_{2}}}+O(1) .
$$

Note that

$$
\begin{aligned}
\Theta_{x_{1} x_{1}}\left(0, x_{2}, \pm 1,0\right) \Theta\left(0, x_{2},\right. & \pm 1,0)-2 \Theta_{x_{1}}\left(0, x_{2}, \pm 1,0\right) \Theta_{x_{1}}\left(0, x_{2}, \pm 1,0\right) \\
& =\left(2 \Theta^{3} r_{x_{1}} r_{x_{1}}-\Theta^{2} r_{x_{1} x_{1}}\right) \Theta-2 \Theta^{2} r_{x_{1}} \Theta^{2} r_{x_{1}}=0,
\end{aligned}
$$

since $r_{x_{1} x_{1}}=0$, and analogously

$$
\Theta_{x_{1} x_{2}}\left(0, x_{2}, \pm 1,0\right) \Theta\left(0, x_{2}, \pm 1,0\right)-2 \Theta_{x_{1}}\left(0, x_{2}, \pm 1,0\right) \Theta_{x_{2}}\left(0, x_{2}, \pm 1,0\right)
$$

Then from (13), (29), (37), and (38) we get

$$
\begin{array}{r}
\Theta_{x_{2} x_{2}}\left(0, x_{2}, \pm 1,0\right) \Theta\left(0, x_{2}, \pm 1,0\right)-2 \Theta_{x_{2}}\left(0, x_{2}, \pm 1,0\right) \Theta_{x_{2}}\left(0, x_{2}, \pm 1,0\right) \\
\Theta\left(0, x_{2}, \pm 1,0\right)^{3} \\
=\frac{1}{2 x_{2}^{3 / 2}}+\frac{2 f^{(4)}(0)}{\sqrt{x_{2}}}+O(1) .
\end{array}
$$

Finally, using (30), (12), (39), (40), (41), (42), and (43), we obtain the series expansions of the metric tensor of the Hilbert metric.

$$
\begin{aligned}
& g_{11}\left(0, x_{2}, 1,0\right)=\frac{1}{4 x_{2}}+O(1), \\
& g_{12}\left(0, x_{2}, 1,0\right)=\frac{f^{\prime \prime \prime}(0)}{6 x_{2}}+O(1), \\
& g_{22}\left(0, x_{2}, 1,0\right)=\frac{1}{4 x_{2}^{2}}+\frac{2 f^{\prime \prime \prime}(0)^{2}+9 f^{(4)}(0)}{18 x_{2}}+O(1) .
\end{aligned}
$$

3.2. Expansions for $\boldsymbol{g}_{i j}\left(\mathbf{0}, \boldsymbol{x}_{\mathbf{2}}, \mathbf{0}, \mathbf{1}\right)$. The formulae in (16) imply that, at $\left(0, x_{2}\right)$,

$$
\begin{aligned}
t_{x_{1}}\left(0, x_{2}, 0, \pm 1\right) & =0 \\
t_{x_{2}}\left(0, x_{2}, 0, \pm 1\right) & =-1 \\
t_{x_{1} x_{2}}\left(0, x_{2}, 0, \pm 1\right) & =t_{x_{2} x_{2}}\left(0, x_{2}, 0, \pm 1\right)=0 .
\end{aligned}
$$

Note that the functions $t\left(0, x_{2}, 0, \pm 1\right)$ have the representations

$$
t\left(0, x_{2}, 0,-1\right)=-x_{2}, \quad t\left(0, x_{2}, 0,1\right)=H-x_{2} .
$$

Here $H$ denotes the length of the chord of $\partial U$ in the direction $(0,1)$. Then

$$
\Theta\left(0, x_{2}, 0,-1\right)=\frac{1}{x_{2}}, \quad \Theta\left(0, x_{2}, 0,1\right)=\frac{1}{H-x_{2}} .
$$


Consequently,

$$
\mathrm{F}\left(0, x_{2}, 0,1\right)=\frac{1}{2}\left(\frac{1}{H-x_{2}}+\frac{1}{x_{2}}\right)=\frac{1}{2 x_{2}}+O(1) .
$$

We can estimate the derivatives of the Funk metrics $\Theta\left(0, x_{2}, 0, \pm 1\right)$. It follows from (19) and (20) that

$$
\begin{aligned}
\Theta_{x_{2}}\left(0, x_{2}, 0,-1\right) & =\frac{1}{x_{2}^{2}}, \quad \Theta_{x_{2}}\left(0, x_{2}, 0,1\right)=-\frac{1}{\left(H-x_{2}\right)^{2}}, \\
\Theta_{x_{2} x_{2}}\left(0, x_{2}, 0,-1\right) & =\frac{2}{x_{2}^{3}}, \quad \Theta_{x_{2} x_{2}}\left(0, x_{2}, 0,1\right)=\frac{2}{\left(H-x_{2}\right)^{3}} .
\end{aligned}
$$

Using (12), (46), and (47), we get the expansions:

$$
\begin{aligned}
& g_{12}\left(0, x_{2}, 0,1\right)=0 \\
& g_{22}\left(0, x_{2}, 0,1\right)=\frac{1}{4}\left(\frac{1}{H-x_{2}}+\frac{1}{x_{2}}\right)^{2}=\frac{1}{4 x_{2}^{2}}+O\left(\frac{1}{x_{2}}\right) .
\end{aligned}
$$

We will also need the values $\mathrm{F}\left(0, x_{2}, l, \frac{1}{2}\right)$.

We have

$$
\begin{aligned}
t\left(0, x_{2},-l,-\frac{1}{2}\right) & =-2 x_{2}+2 l^{2} x_{2}^{2}+O\left(x_{2}^{3}\right), \\
t\left(0, x_{2}, l, \frac{1}{2}\right) & =L+O\left(x_{2}\right) .
\end{aligned}
$$

Then

$$
\begin{aligned}
\mathrm{F}\left(0, x_{2}, l, \frac{1}{2}\right)= & \frac{\sqrt{\frac{1}{4}+l^{2}}}{2 \sqrt{\frac{1}{4} t\left(0, x_{2}, l, \frac{1}{2}\right)^{2}+\left(l t\left(0, x_{2}, l, \frac{1}{2}\right)\right)^{2}}} \\
& +\frac{\sqrt{\frac{1}{4}+l^{2}}}{2 \sqrt{\frac{1}{4} t\left(0, x_{2},-l,-\frac{1}{2}\right)^{2}+\left(l t\left(0, x_{2},-l,-\frac{1}{2}\right)\right)^{2}}} \\
= & \frac{1}{2 \sqrt{\frac{1}{4}+l^{2}}}\left(\frac{1}{t\left(0, x_{2}, l, \frac{1}{2}\right)}-\frac{1}{t\left(0, x_{2},-l,-\frac{1}{2}\right)}\right) .
\end{aligned}
$$

Finally,

$$
\mathrm{F}\left(0, x_{2}, l, \frac{1}{2}\right)=\frac{1}{4 x_{2}}+\frac{1}{2 L}+O\left(x_{2}\right) .
$$

\section{Proof of the theorems}

The Chern-Rund covariant derivative along the curve $c(t)$ in the Finsler space equipped with the Hilbert metric $F$ is given by the formula [Shen 2001]

$$
\nabla_{c^{\prime}(t)} c^{\prime}(t)=\left\{c^{\prime \prime}(t)^{i}+\left(\Theta\left(c(t), c^{\prime}(t)\right)-\Theta\left(c(t),-c^{\prime}(t)\right) c^{\prime}(t)^{i}\right\} \frac{\partial}{\partial x^{i}} .\right.
$$


For calculating the normal curvature (2), the Finsler curvature (3), and the Rund curvature (4), we need the covariant derivative $\nabla_{\dot{c}(s)} \dot{c}(s)$ of the curve $c(s)$ parametrized by its arc length.

For a given curve $c(t)$, we will denote by the dot the derivative with respect to the arc length $s$, and by the prime the derivative with respect to $t$. Then let $t=t(s)$ be the reparametrization. We get

$$
\dot{c}(s)=c^{\prime}(t) t_{s}^{\prime} .
$$

Using that $s$ in the length parameter, we get

$$
1=\mathrm{F}\left(c(t), c^{\prime}(t)\right) t_{s}^{\prime} .
$$

Hence

$$
\dot{c}(s)=\frac{c^{\prime}(t)}{\mathrm{F}\left(c(t), c^{\prime}(t)\right)} .
$$

The next step is to calculate $\nabla_{\dot{c}(s)} \dot{c}(s)$.

$$
\begin{aligned}
\nabla_{\dot{c}(s)} \dot{c}(s) & =\nabla_{c^{\prime}(t) / \mathrm{F}\left(c(t), c^{\prime}(t)\right)} \frac{c^{\prime}(t)}{\mathrm{F}\left(c(t), c^{\prime}(t)\right)} \\
& =\frac{1}{\mathrm{~F}\left(c(t), c^{\prime}(t)\right)}\left(\nabla_{c^{\prime}(t)}\left(\frac{1}{\mathrm{~F}\left(c(t), c^{\prime}(t)\right)}\right) c^{\prime}(t)+\frac{1}{\mathrm{~F}\left(c(t), c^{\prime}(t)\right)} \nabla_{c^{\prime}(t)} c^{\prime}(t)\right) .
\end{aligned}
$$

According to [Bao et al. 2000],

$$
\nabla_{c^{\prime}(t)}\left(\frac{1}{\mathrm{~F}\left(c(t), c^{\prime}(t)\right)}\right)=-\frac{\boldsymbol{g}_{c^{\prime}(t)}\left(\nabla_{c^{\prime}(t)} c^{\prime}(t), c^{\prime}(t)\right)}{\mathrm{F}\left(c(t), c^{\prime}(t)\right)^{3}} .
$$

Then the derivative $\nabla_{\dot{c}(s)} \dot{c}(s)$ has the form

$$
\nabla_{\dot{c}(s)} \dot{c}(s)=\frac{1}{\mathrm{~F}\left(c(t), c^{\prime}(t)\right)^{2}}\left(\nabla_{c^{\prime}(t)} c^{\prime}(t)-\frac{\boldsymbol{g}_{c^{\prime}(t)}\left(\nabla_{c^{\prime}(t)} c^{\prime}(t), c^{\prime}(t)\right)}{\mathrm{F}\left(c(t), c^{\prime}(t)\right)^{2}} c^{\prime}(t)\right) .
$$

Finally, using (50), we get the formula:

$$
\begin{aligned}
& \nabla_{\dot{c}(s)} \dot{c}(s)= \\
& \frac{c^{\prime \prime}(t)+c^{\prime}(t)\left(\Theta\left(c(t), c^{\prime}(t)\right)-\Theta\left(c(t),-c^{\prime}(t)\right)-\frac{\boldsymbol{g}_{c^{\prime}(t)}\left(\nabla_{c^{\prime}(t)} c^{\prime}(t), c^{\prime}(t)\right)}{\mathrm{F}\left(c(t), c^{\prime}(t)\right)^{2}}\right)}{\mathrm{F}\left(c(t), c^{\prime}(t)\right)^{2}} .
\end{aligned}
$$

As in Section 2 fix a point $o$ in the domain $U$ and a point $p \in \partial U$. The curve $\partial U$ admits the polar representation $\omega(\varphi)$ from the point $o$ such that the point $p$ corresponds to $\varphi=0$. According to Section 2, we assume that $U$ satisfies the conditions (1)-(4). 
Then one can get that $\omega^{\prime}(0)=0, \omega(0)=1, \omega^{\prime \prime}(0)=\frac{1}{2}, \omega^{\prime}(\pi)=0$. Set

$$
C=\frac{1+\omega(\pi)}{\omega(\pi)} .
$$

In [Borisenko and Olin 2008] the polar function $\rho_{r}(u)$ of the hypersphere of radius $r$ was obtained:

$$
\rho_{r}(u)=\frac{\omega(-u) \omega(u)\left(e^{2 r}-1\right)}{\omega(u)+\omega(-u) e^{2 r}} .
$$

As $r \rightarrow \infty$,

$$
\omega(u)-\rho_{r}(u)=\omega(u)\left(\frac{\omega(u)}{\omega(-u)}+1\right) e^{-2 r}+o\left(e^{-2 r}\right) .
$$

From (52) we get that the circle of radius $r$ admits the parametrization

$$
c(\varphi)=\left(\frac{\omega(\pi-\varphi) \omega(\varphi)\left(e^{2 r}-1\right)}{\omega(\varphi)+\omega(\pi-\varphi) e^{2 r}} \sin \varphi, \frac{\omega(\pi-\varphi) \omega(\varphi)\left(e^{2 r}-1\right)}{\omega(\varphi)+\omega(\pi-\varphi) e^{2 r}} \cos \varphi\right),
$$

where $\omega(\varphi)$ is the polar function of $\partial U$.

Then

$$
c^{\prime}(0)=\frac{\omega(\pi)\left(e^{2 r}-1\right)}{1+\omega(\pi) e^{2 r}}(1,0)=\left(1-C e^{-2 r}+O\left(e^{-3 r}\right), 0\right), \quad r \rightarrow \infty .
$$

The second derivative:

$$
\begin{aligned}
& c^{\prime \prime}(0)=\frac{\left(e^{2 r} \omega(\pi)^{2}\left(\omega^{\prime \prime}(0)-1\right)-\omega(\pi)+\omega^{\prime \prime}(\pi)\right)\left(e^{2 r}-1\right)}{\left(1+e^{2 r} \omega(\pi)\right)^{2}}(0,1), \\
& c^{\prime \prime}(0)=\left(0,-\frac{1}{2}+O\left(e^{-2 r}\right)\right), \quad r \rightarrow \infty .
\end{aligned}
$$

From (53) we get that at the point of the circle the second coordinate is

$$
x_{2}=\omega(0)-\frac{\omega(\pi) \omega(0)\left(e^{2 r}-1\right)}{\omega(0)+\omega(\pi) e^{2 r}}=C e^{-2 r}+O\left(e^{-3 r}\right) .
$$

Estimate the derivative $\nabla_{\dot{c}(0)} \dot{c}(0)$ using the formulae (51), (31), and (56):

$$
\begin{aligned}
\Theta\left(c(0), c^{\prime}(0)\right) & -\Theta\left(c(0),-c^{\prime}(0)\right)=\Theta\left(0, C e^{-2 r}+O\left(e^{-3 r}\right), 1+O\left(e^{-2 r}\right), 0\right) \\
& -\Theta\left(0, C e^{-2 r}+O\left(e^{-3 r}\right),-1+O\left(e^{-2 r}\right), 0\right)=\frac{2}{3} f^{\prime \prime \prime}(0)+O\left(e^{-2 r}\right) .
\end{aligned}
$$

Therefore, formula (50) leads to

$$
\begin{aligned}
\nabla_{c^{\prime}(0)} c^{\prime}(0) & =c^{\prime \prime}(0)+c^{\prime}(0)\left(\Theta\left(c(0), c^{\prime}(0)\right)-\Theta\left(c(0),-c^{\prime}(0)\right)\right) \\
& =\left(\frac{2}{3} f^{\prime \prime \prime}(0),-\frac{1}{2}\right)+O\left(e^{-2 r}\right) .
\end{aligned}
$$


Using (56) and (57) we get

$$
\frac{\boldsymbol{g}_{c^{\prime}(0)}\left(\nabla_{c^{\prime}(0)} c^{\prime}(0), c^{\prime}(0)\right)}{\mathrm{F}\left(c(0), c^{\prime}(0)\right)^{2}}=\frac{\frac{2}{3} f^{\prime \prime \prime}(0) g_{11}-\frac{1}{2} g_{12}}{\mathrm{~F}\left(0, C e^{-2 r}+O\left(e^{-3 r}\right), 1+O\left(e^{-2 r}\right), 0\right)^{2}} .
$$

Here $g_{i j}$ are calculated at the point $\left(0, C e^{-2 r}+O\left(e^{-3 r}\right), 1+O\left(e^{-2 r}\right), 0\right)$. After substituting the values from (30) and (44), we obtain

$$
\frac{\boldsymbol{g}_{c^{\prime}(0)}\left(\nabla_{c^{\prime}(0)} c^{\prime}(0), c^{\prime}(0)\right)}{\mathrm{F}\left(c(0), c^{\prime}(0)\right)^{2}}=-\frac{f^{\prime \prime \prime}(0)}{3}+O\left(e^{-2 r}\right) .
$$

Therefore,

$$
\nabla_{\dot{c}(0)} \dot{c}(0)=\frac{\left(f^{\prime \prime \prime}(0),-\frac{1}{2}\right)+(1,1) O\left(e^{-2 r}\right)}{\mathrm{F}\left(c(0), c^{\prime}(0)\right)^{2}} .
$$

Taking into account (30),

$$
\nabla_{\dot{c}(0)} \dot{c}(0)=\left(4 f^{\prime \prime \prime}(0),-2\right) e^{-2 r}+(1,1) O\left(e^{-3 r}\right) .
$$

Calculate the Rund curvature (4) using the formulae (56) and (58).

$$
\begin{aligned}
\boldsymbol{k}_{R}(r)^{2} & =\mathrm{F}\left(c(0), \nabla_{\dot{c}(0)} \dot{c}(0)\right) \\
& =\frac{\mathrm{F}\left(0, C e^{-2 r}+O\left(e^{-3 r}\right),-f^{\prime \prime \prime}(0)+O\left(e^{-2 r}\right), \frac{1}{2}+O\left(e^{-2 r}\right)\right)}{\mathrm{F}\left(0, C e^{-2 r}+O\left(e^{-3 r}\right), 1-C e^{-2 r}+O\left(e^{-3 r}\right), 0\right)^{2}} .
\end{aligned}
$$

From (30) and (49) we get

$$
\boldsymbol{k}_{R}(r)^{2}=1+C\left(\frac{2}{L}-\frac{8 f^{\prime \prime \prime}(0)^{2}}{9}\right) e^{-2 r}+O\left(e^{-3 r}\right) .
$$

Here $L>0$ is the length of the chord $\ell$ of $\partial U$ in the direction $\left(f^{\prime \prime \prime}(0),-1 / 2\right)$. Proposition 2.2 gives the uniform bounds on the curvature of $\partial U$. Proposition 2.3 claims that the angle between the chord $\ell$ and $x_{2}$ is uniformly separated from $\pi / 2$. Thus we conclude that $2 / L$ is bounded from above.

Calculate the Finsler curvature (3) using the formulae (56) and (58).

$$
\begin{aligned}
\boldsymbol{k}_{F}(r)^{2} & =\boldsymbol{g}_{\dot{c}(0)}\left(\nabla_{\dot{c}(0)} \dot{c}(0), \nabla_{\dot{c}(0)} \dot{c}(0)\right) \\
& =\frac{f^{\prime \prime \prime}(0)^{2} g_{11}-f^{\prime \prime \prime}(0) g_{12}+\frac{1}{4} g_{22}}{\mathrm{~F}\left(0, C e^{-2 r}+O\left(e^{-3 r}\right), 1-C e^{-2 r}+O\left(e^{-3 r}\right), 0\right)^{4}} .
\end{aligned}
$$

Here $g_{i j}$ are considered at the point $\left(0, C e^{-2 r}+O\left(e^{-3 r}\right), 1+O\left(e^{-2 r}\right), 0\right)$. Finally, from (30) and (44) we obtain that

$$
\boldsymbol{k}_{F}(r)^{2}=1+C\left(-\frac{8}{9} f^{\prime \prime \prime}(0)^{2}+4 f^{(4)}(0)\right) e^{-2 r}+O\left(e^{-3 r}\right) .
$$

Proposition 2.3 gives the uniform bounds on the derivatives of $f$. Theorem 1.1 is proved. 
Note that the normal curvature $\boldsymbol{g}_{\boldsymbol{n}}\left(\nabla_{\dot{c}(s)} \dot{\boldsymbol{c}}(s), \boldsymbol{n}\right)$ of a hypersurface at the point $x$ depends only on the tangent vector to the curve $c(s)$ at $x$ [Shen 2001]. So in order to obtain the normal curvature of the Hilbert hypersphere $S_{r}$ centered at $o$ at the point $p$ in the tangent direction $w$, we consider the normal curvature of the circle $S_{r} \cap \Pi$ which lies in the plane $\Pi=\operatorname{span}(w, \overrightarrow{o p})$.

From (57) we get the normal curvature of the circle of radius $r$ :

$$
\boldsymbol{k}_{\boldsymbol{n}}(r)=\boldsymbol{g}_{\boldsymbol{n}}\left(\nabla_{\dot{c}(0)} \dot{c}(0), \boldsymbol{n}\right)=\frac{\boldsymbol{g}_{\boldsymbol{n}}\left(c^{\prime \prime}(0), \boldsymbol{n}\right)}{\mathrm{F}\left(c(0), c^{\prime}(0)\right)^{2}} .
$$

Since $g_{12}\left(0, x_{2}, 0,1\right)=0$ by (48), it follows that the unit normal vector $\boldsymbol{n}$ to the circle at $\left(0, x_{2}\right)$ is exactly

$$
\frac{1}{\mathrm{~F}\left(0, x_{2}, 0,1\right)}(0,-1) \text {. }
$$

Finally, taking into account (30), (56), (55), (45), and (48):

$$
\begin{aligned}
& \boldsymbol{k}_{\boldsymbol{n}}(r) \\
= & \frac{\frac{1}{2} g_{22}\left(0, C e^{-2 r}+O\left(e^{-3 r}\right), 0,1\right)}{\mathrm{F}\left(0, C e^{-2 r}+O\left(e^{-3 r}\right), 1-C e^{-2 r}+O\left(e^{-3 r}\right), 0\right)^{2} \mathrm{~F}\left(0, C e^{-2 r}+O\left(e^{-3 r}\right), 0,1\right)} \\
= & 1+C\left(\frac{1}{H}-\frac{8 f^{\prime \prime \prime}(0)^{2}}{9}\right) e^{-2 r}+O\left(e^{-3 r}\right) .
\end{aligned}
$$

If it is the case that the Euclidean normal curvatures of the hypersurface $\partial U$ are bounded $\left(k_{2} \leqslant k_{n} \leqslant k_{1}\right)$ then the curvature of the curve $\partial U^{\prime}=\partial U \cap \Pi$ is bounded as well. Consider the point $x \in \partial U^{\prime} \subset \partial U$. Then the curvature $k(x)$ of $\partial U^{\prime}$ and the normal curvature $k_{n}(x)$ of $\partial U$ are related as

$$
k(x)=\frac{k_{n}(x)}{\cos \beta} .
$$

Here $\beta$ is the angle between the radial and normal direction to $\partial U$ at $x$. Using Lemma 2.1 we find that $\omega_{0} k_{2} \leqslant \cos \beta \leqslant 1$. Hence the curvature of $\partial U^{\prime}$ is uniformly bounded for all $y$. Applying Proposition 2.2 for the Hilbert geometry based on $U^{\prime}$, we get the uniformity of the series expansion (62) which ends the proof of Theorem 1.2.

\section{References}

[Bao et al. 2000] D. Bao, S.-S. Chern, and Z. Shen, An introduction to Riemann-Finsler geometry, Graduate Texts in Mathematics 200, Springer, New York, 2000. MR 2001g:53130 Zbl 0954.53001 [Borisenko 2002] A. A. Borisenko, "Convex sets in Hadamard manifolds", Differential Geom. Appl. 17:2-3 (2002), 111-121. MR 2003k:53035 Zbl 1034.53056

[Borisenko and Olin 2008] A. A. Borisenko and E. A. Olin, "Asymptotic properties of Hilbert geometry”, Zh. Mat. Fiz. Anal. Geom. 4:3 (2008), 327-345, 443. MR 2010e:53124 Zbl 1167.53066 
[Colbois and Verovic 2002] B. Colbois and P. Verovic, "Rigidity of Hilbert metrics", Bull. Austral. Math. Soc. 65:1 (2002), 23-34. MR 2003c:53053 Zbl 1007.53055

[Finsler 1951] P. Finsler, Über Kurven und Flächen in allgemeinen Räumen, Birkhäuser, Basel, 1951. MR 13,74d Zbl 0044.37003

[Rund 1959] H. Rund, The differential geometry of Finsler spaces, Die Grundlehren der Mathematischen Wissenschaften 101, Springer, Berlin, 1959. MR 21 \#4462 Zbl 0087.36604

[Shen 2001] Z. Shen, Lectures on Finsler geometry, World Scientific, Singapore, 2001. MR 2002f: 53032 Zbl 0974.53002

Received May 10, 2011.

ALEXANDER BORISENKO

School of Mathematics and Mechanical Engineering, Geometry Department KHARKOV NATIONAL UNIVERSITY

SVOBODY SQUARE, 4

KHARKOV 61022

UKRAINE

borisenk@univer.kharkov.ua

EUGENE OLIN

School of Mathematics and Mechanical Engineering, Geometry Department KHARKOV NATIONAL UNIVERSITY

SVOBODY SQUARE, 4

KHARKOV 61022

UKRAINE

evolin@mail.ru

http://geometry.univer.kharkov.ua/ olin/olin.html 


\title{
PACIFIC JOURNAL OF MATHEMATICS
}

\author{
http://pacificmath.org \\ Founded in 1951 by \\ E. F. Beckenbach (1906-1982) and F. Wolf (1904-1989)
}

\section{EDITORS}

V. S. Varadarajan (Managing Editor)

Department of Mathematics

University of California

Los Angeles, CA 90095-1555

pacific@math.ucla.edu

Vyjayanthi Chari

Department of Mathematics

University of California

Riverside, CA 92521-0135

chari@math.ucr.edu

\section{Robert Finn}

Department of Mathematics Stanford University

Stanford, CA 94305-2125

finn@math.stanford.edu

Kefeng Liu

Department of Mathematics

University of California

Los Angeles, CA 90095-1555

liu@math.ucla.edu
Darren Long

Department of Mathematics

University of California

Santa Barbara, CA 93106-3080

long@math.ucsb.edu

Jiang-Hua Lu

Department of Mathematics

The University of Hong Kong

Pokfulam Rd., Hong Kong jhlu@maths.hku.hk

Alexander Merkurjev

Department of Mathematics

University of California

Los Angeles, CA 90095-1555

merkurev@math.ucla.edu
Sorin Popa

Department of Mathematics University of California

Los Angeles, CA 90095-1555 popa@math.ucla.edu

Jie Qing

Department of Mathematics

University of California

Santa Cruz, CA 95064

qing@cats.ucsc.edu

Jonathan Rogawski

Department of Mathematics

University of California

Los Angeles, CA 90095-1555

jonr@math.ucla.edu

\section{PRODUCTION}

pacific@math.berkeley.edu

\section{SUPPORTING INSTITUTIONS}

ACADEMIA SINICA, TAIPEI

CALIFORNIA INST. OF TECHNOLOGY INST. DE MATEMÁTICA PURA E APLICADA KEIO UNIVERSITY

MATH. SCIENCES RESEARCH INSTITUTE NEW MEXICO STATE UNIV.

OREGON STATE UNIV.

\author{
STANFORD UNIVERSITY \\ UNIV. OF BRITISH COLUMBIA \\ UNIV. OF CALIFORNIA, BERKELEY \\ UNIV. OF CALIFORNIA, DAVIS \\ UNIV. OF CALIFORNIA, LOS ANGELES \\ UNIV. OF CALIFORNIA, RIVERSIDE \\ UNIV. OF CALIFORNIA, SAN DIEGO \\ UNIV. OF CALIF., SANTA BARBARA
}

\author{
UNIV. OF CALIF., SANTA CRUZ \\ UNIV. OF MONTANA \\ UNIV. OF OREGON \\ UNIV. OF SOUTHERN CALIFORNIA \\ UNIV. OF UTAH \\ UNIV. OF WASHINGTON \\ WASHINGTON STATE UNIVERSITY
}

These supporting institutions contribute to the cost of publication of this Journal, but they are not owners or publishers and have no responsibility for its contents or policies.

See inside back cover or pacificmath.org for submission instructions.

The subscription price for 2011 is US \$420/year for the electronic version, and \$485/year for print and electronic.

Subscriptions, requests for back issues from the last three years and changes of subscribers address should be sent to Pacific Journal of Mathematics, P.O. Box 4163, Berkeley, CA 94704-0163, U.S.A. Prior back issues are obtainable from Periodicals Service Company, 11 Main Street, Germantown, NY 12526-5635. The Pacific Journal of Mathematics is indexed by Mathematical Reviews, Zentralblatt MATH, PASCAL CNRS Index, Referativnyi Zhurnal, Current Mathematical Publications and the Science Citation Index.

The Pacific Journal of Mathematics (ISSN 0030-8730) at the University of California, c/o Department of Mathematics, 969 Evans Hall, Berkeley, CA 94720-3840, is published monthly except July and August. Periodical rate postage paid at Berkeley, CA 94704, and additional mailing offices. POSTMASTER: send address changes to Pacific Journal of Mathematics, P.O. Box 4163, Berkeley, CA 94704-0163.

PJM peer review and production are managed by EditFLOW ${ }^{\mathrm{TM}}$ from Mathematical Sciences Publishers.

PUBLISHED BY PACIFIC JOURNAL OF MATHEMATICS

at the University of California, Berkeley 94720-3840

A NON-PROFIT CORPORATION

Typeset in LATEX

Copyright $(2011$ by Pacific Journal of Mathematics 


\section{PACIFIC JOURNAL OF MATHEMATICS}

Volume $254 \quad$ No. $2 \quad$ December 2011

Curvatures of spheres in Hilbert geometry

ALEXANDER BORISENKO and EUGENE OLIN

A formula equating open and closed Gromov-Witten invariants and its applications 275

to mirror symmetry

KWOKWAI CHAN

A note on $p$-harmonic $l$-forms on complete manifolds

LiAng-CHu CHANG and CHIUng-JUe ANNA SUNG

The Cheeger constant of curved strips

DAVID KREJČIŘíK and ALdo PRATELLI

Structure of solutions of 3D axisymmetric Navier-Stokes equations near maximal points

\section{ZHEN LEI and QI S. ZHANG}

Local comparison theorems for Kähler manifolds

GANG LIU

Structurable algebras of skew-rank 1 over the affine plane

SUSANNE PUMPLÜN

An analogue of Krein's theorem for semisimple Lie groups

SANJOY PUSTI

Une remarque de dynamique sur les variétés semi-abéliennes

GAËL RÉMOND

Fourier transforms of semisimple orbital integrals on the Lie algebra of $\mathrm{SL}_{2}$

LOREN SPICE

On noncompact $\tau$-quasi-Einstein metrics

LIN FENG WANG

Decomposition of de Rham complexes with smooth horizontal coefficients for semistable reductions

QIHONG XIE

A differentiable sphere theorem inspired by rigidity of minimal submanifolds 